\title{
The effect of probiotics on functional constipation: a systematic review of randomised controlled trials
}

\author{
E. Dimidi ${ }^{1}$, S. Christodoulides ${ }^{2}$, K. C. Fragkos ${ }^{3}$, S. M. Scott ${ }^{2}$ and K. Whelan ${ }^{1}$ \\ ${ }^{1}$ Diabetes and Nutritional Sciences Division, King's College London, 150 Stamford Street, London, SE1 9NH, \\ ${ }^{2}$ Centre for Digestive Diseases, Queen Mary University of London, 26 Ashfield Street, London, E1 2 A and \\ ${ }^{3}$ Centre for Gastroenterology and Clinical Nutrition, Division of Medicine, University College London, \\ Rockefeller Building, 21 University Street, London, WC1E 6DE
}

Constipation is characterised by infrequent stools and/or difficulty in stool passage and has a prevalence of approximately $14 \%$ in adults ${ }^{(1)}$. Several management strategies are currently used including laxatives, bulking agents and stool softeners but many patients are dissatisfied with these due to variable efficacy ${ }^{(2)}$. Probiotics are live microorganisms that, when administered in adequate amounts, confer a health benefit to the host. Some probiotics increase short-chain fatty acids in the large intestine, resulting in increased propulsive contractions, which may result in reduced gut transit time ${ }^{(3)}$. There has been increasing research regarding the effect of probiotics in constipation, and a systematic review is required in order to evaluate the quality and results of these studies.

The aim of this study was to investigate the effect of probiotics in functional constipation via a systematic review of randomised controlled trials (RCTs) in adults.

This review followed the Cochrane and PRISMA recommendations. Studies were identified by searching four electronic databases, scanning reference lists, contacting authors, and hand searching of abstracts of eight annual conferences. RCTs reporting administration of probiotics in adults with functional constipation or constipation-predominant irritable bowel syndrome were included in the review. Two reviewers independently performed the screening of articles, data extraction, and risk of bias assessment. Disagreements were resolved by a third team member.

The abstracts of 656 articles were screened, of which 61 articles were reviewed in full. Thirteen RCTs were eligible (including 1,347 patients). Probiotics were administered in the form of fermented milk, yoghurt, drinks, capsules, tablets, sachets or probiotic-fortified food. The probiotics were Bifidobacterium lactis $(n=6)$, B. animalis $(n=2)$, B. breve $(n=1)$, Lactobacillus casei $(n=3)$, L. reuteri $(n=1)$, L. plantarum $(n=1)$, L. paracasei $(n=1)$ and Escherichia coli Nissle $1917(n=1)$. The outcomes measured included whole gut transit time (WGTT; $n=3)$, stool frequency $(n=13)$, stool consistency $(n=8)$, adverse events $(n=7)$, compliance $(n=3)$ and tolerability $(n=2)$. Two out of three RCTs reported a significant lower WGTT following probiotic consumption compared to placebo. Stool frequency was significantly higher following probiotic consumption in 7 RCTs, while 6 RCTs failed to show a significant effect. Four RCTs demonstrated a significant beneficial difference in stool consistency, but 4 RCTs did not. The study products were well tolerated and compliance rates varied from $95 \%-100 \%$. No adverse events were reported in 5 RCTs, whilst one reported minor adverse events (bloating, loose stools, flu symptoms) and in another, three subjects in the probiotic group and two in the placebo group ceased product consumption following adverse events. Attrition and reporting bias were high amongst the trials, while selection bias was unclear due to inadequate reporting. Outcome data will undergo meta-analysis.

Though evidence of the effect of probiotics on functional constipation is inconsistent, probiotics seem to have beneficial effects on symptoms of constipation, and are associated with low risk of adverse events. Their benefits on constipation may occur directly or indirectly from their effect on the composition and/or activity of the gut microbiota, and/or stimulation of the immune system. This systematic review emphasizes the need for good quality RCT investigating the effect of probiotic on gastrointestinal health in adults with functional constipation.

1. Suares NC, Ford AC (2011) Am. J. Gastroenterol 106, 1582-91.

2. Johanson JF \& Kralstein J (2007) Aliment Pharmacol Ther 25, 599-608

3. Yajima T (1985) J Physiol 368, 667-78. 